\title{
Hypersingular integral equations for multiple interacting planar cracks in an elastic layered material under antiplane shear stresses
}

\author{
W. T. Ang \\ Faculty of Engineering, Universiti Malaysia Sarawak (UNIMAS), 94300 Kota Samarahan, Malaysia \\ $\&$ \\ D. L. Clements \\ Department of Applied Mathematics, University of Adelaide, Adelaide, South Australia 5005, Australia
}

(Received 20 May 1995; accepted 15 September 1995)

\begin{abstract}
A system of Hadamard finite-part singular (hypersingular) integral equations is derived for an antiplane elastic problem involving an arbitrary number of arbitrarily-located planar cracks in a tri-layered material. The equations are solved approximately to compute the stress intensity factors for specific cases of the problems.
\end{abstract}

Key words: Hadamard finite-part singular integral equations, multiple planar cracks, antiplane deformations, numerical solution.

\section{INTRODUCTION}

Many two-dimensional crack problems in linear elasticity can be reduced to solving systems of Hadamard finite-part singular (hypersingular) integral equations of the form

$$
\begin{gathered}
\mathcal{H} \int_{-1}^{1}(u-s)^{-2} r_{j}(u) \mathrm{d} u+\sum_{k=1}^{N} \int_{-1}^{1} T_{j k}(u, s) r_{k}(u) \mathrm{d} u \\
=P_{j}(s) \text { for }-1<s<1(j=1,2, \ldots, N)
\end{gathered}
$$

where $\mathcal{H}$ denotes that the integral over the given interval should be interpreted in the Hadamard finite-part sense, $T_{j k}(u, s)$ and $P_{j}(s)$ are known functions and $r_{k}(u)$ are unknown functions which are directly related to the crack opening displacements. The Hadamard finitepart integral arises because of the differentiation of a Cauchy principle integral which appears in the expression for the displacement at points on the crack surfaces.

Recently, with the availability of effective numerical methods for solving hypersingular integral equations, such as the collocation technique described in Kaya and Erdogan, ${ }^{1}$ there has been growing interest among researchers in using such integral equations to formulate crack problems. For example, with the aid of Fourier integral transforms, Nied ${ }^{2}$ obtained Hadamard finitepart singular integral equations for a periodic array of planar cracks in an isotropic half-space, $\mathrm{Ang}^{3}$ for a pair of coplanar cracks in an infinitely long anisotropic strip, and Ang and Noone ${ }^{4}$ for coplanar cracks in a rectangular anisotropic slab. Using suitable boundary integral equations and Green's functions, Ang et al..$^{5-7}$ formulated various two-dimensional crack problems concerning an arbitrary number of arbitrarily located planar cracks in terms of hypersingular integral equations. Earlier work on the hypersingular integral formulation of crack problems may be found in Ioakimidis. $^{8}$

In the present paper, we consider the problem of multiple interacting planar cracks in an elastic strip sandwiched between two elastic half-spaces. Clements ${ }^{9}$ studied this problem for the particular case of a singular planar crack lying on a plane parallel to the interfaces separating the strip and the half-spaces. The case where the planar crack is located on a plane which is perpendicular to the interfaces was later examined by Ang. ${ }^{10}$ The method of solution used in Refs 9 and 10, based on the theory of dual integrals, cannot be readily modified to take into consideration cases involving multiple interacting cracks. Starting with a suitable integral 